Luigi BISIO*

\title{
Terzo contributo alla conoscenza di Oreonebria (Nebriorites) gagates (Bonelli, 1810): una sintesi (Coleoptera Carabidae)
}

\begin{abstract}
Riassunto: Viene descritta l'ecologia di Oreonebria (Nebriorites) gagates (Bonelli, 1810). La specie vive principalmente nelle fenditure tra grandi blocchi di roccia di paleofrane o di antiche morene. La sua presenza all'interno di questi litosuoli è probabilmente da mettere in relazione alle sue peculiari esigenze ecologiche che la portano a ricercare biotopi caratterizzati da fattori abiotici costanti con umidità elevata e basse temperature. Viene anche descritta la metodologia utilizzata per la ricerca della specie in questi substrati: essa si basa sull'uso di trappole costituite da bottiglie innescate con aceto di vino (addizionato con acido salicilico a scopo conservante) che devono essere collocate, per quanto possibile, nelle zone più profonde all'interno delle fessure, appoggiate in posizione inclinata sulla superficie dei blocchi di roccia. Viene inoltre fornita un'analisi della sua corologia lungo il versante italiano delle Alpi Occidentali: O. gagates è diffusa dalla Valle Po (Alpi Cozie) alla Valle Anzasca (Alpi Pennine). Infine, sono riassunte le diverse opinioni sulla tassonomia e sulla sistematica della specie espresse da diversi autori e sono elencati i caratteri diagnostici (morfologia e chetotassi del pronoto, morfologia dell'edeago) che, secondo Coulon (1994), permetterebbero di distinguere la ssp. veronicae dalla ssp. nominale. Sulla base di questi caratteri, lungo il versante italiano la sottospecie nominale pare vivere nelle Alpi Cozie, mentre nelle Graie sembra presente la ssp. veronicae. Purtroppo, però, i pochi esemplari disponibili non permettono al momento di verificare la costanza di tali caratteri e di confermare la sua effettiva validità.
\end{abstract}

Abstract: Third Contribution to the knowledge of Oreonebria (Nebriorites) gagates (Bonelli, 1810): a synthesis (Coleoptera Carabidae).

The ecology of Oreonebria (Nebriorites) gagates (Bonelli, 1810) is described. This species lives mainly in the fissures between the rock blocks in the paleo-landslides and in the ancient moraines. Its presence within these lithosoils is probably related to its peculiar ecological requirements which are found in biotopes characterized by constant abiotic factors with high humidity and low temperatures. The method used to sample the species in these substrates is also described: it is based on the use of traps made by bottles triggered with wine vinegar with the addition of the salicylic acid for conservation purposes, which are placed, as far as possible, in the deepest areas within the fissures, in an inclined position on the surface of the rock blocks. An analysis of its chorology along the Italian slope of the Western Alps is provided: $O$. gagates is distributed from the Po Valley (Cottian Alps) to the Anzasca Valley (Pennine Alps). Furthermore, the opinions about the taxonomy and systematics of this species as expressed by different authors are summarized and the diagnostic characters (morphology and chaetotaxy of the pronotum, aedeagus) that would distinguish the ssp. veronicae from the nominal subspecies according to Coulon (1994) are described. Basing on these characters, along the Italian slope of the Western Alps the nominal subspecies seems to be present in the Cottian Alps, while the ssp. veronicae in the Graian Alps, but, unfortunately, at present the few available specimens do not allow to check the constancy of these characters and to confirm its effective validity.

Key words: Coleoptera, Carabidae, Oreonebria, Western Alps, ecology, distributions, taxonomy.

\section{INTRODUZIONE}

L'idea del presente lavoro è nata in seguito al recente rinvenimento da parte dello scrivente di una popolazione di Oreonebria (Nebriorites) gagates (Bonelli, 1810) nella Valle di Champorcher (AO), valle dove la specie era del tutto sconosciuta. L'autore si era occupato già passato di questa entità in due note (Bisio, 1986, 1998), ma da allora diversi nuovi dati sono stati pubblicati. Con il presente lavoro si intende riassumere le conoscenze a tutt'oggi disponibili sull'ecologia, sulla corologia, sulla tassonomia e sulla sistematica di questo interessante endemita delle Alpi
Occidentali (corotipo: W-Alpino, sensu Vigna Taglianti, 2005).

\section{ECOLOGIA}

Alla luce delle attuali conoscenze $O$. gagates pare popolare ambienti molto diversi. Lungo il versante italiano delle Alpi Occidentali essa è considerata soprattutto come un elemento ipogeofilo (sensu Giachino \& Vailati, 2016) che popola i macereti a grandi blocchi (per lo più corpi e conoidi di accumulo di paleofrane $\mathrm{o}$, più in quota, antichi corpi morenici e rock glaciers), situati lungo i pendii meno esposti e nelle

*Luigi Bisio, Via Galilei 4, 10082 Cuorgnè (TO), Italia. E-mail: luigibisio@virgilio.it 
conche più umide e fredde (Figg. 1 e 2 ). In tali substrati essa è spesso rinvenuta in sede microclasica (sensu Brandmayr et al., 1980) negli interstizi tra i grandi massi, preferendo le zone più profonde del macereto che sono meno influenzate dalle temperature esterne. Queste sue abitudini sotterranee si devono probabilmente collegare alle peculiari esigenze ecologiche (igrofilia e frigofilia) che la portano a ricercare biotopi caratterizzati da fattori abiotici costanti (umidità elevata e temperature basse).

O. gagates compare sulla superficie del macereto solo durante la fase di disgelo del manto nevoso che lo ricopre, quando cioè lo stillicidio dell'acqua di fusione mantiene tali condizioni ambientali ottimali anche all'esterno. In questa fase è possibile trovare individui della specie con ricerche a vista, nascosti nelle fessure sature d'acqua tra le pietre accatastate su blocchi più grandi che presentino concavità favorenti il ristagno, anche se occorre evidenziare che tali ricerche non sono sempre fruttuose e, quando lo sono, il risultato è per lo più limitato a pochi esemplari sporadici. Ai margini dei nevai, soprattutto nella fascia alpina, essa entra talvolta in contatto con gli elementi perinivali in senso stretto caratterizzanti il Nebrietum nivale (sensu Focarile, 1973), presenti all'interno del suo areale. Sono stati infatti riscontrati, di volta in volta, casi di sintopia con Oreonebria (Oreonebria) castanea castanea (Bonelli, 1810), Oreonebria (Oreonebria) ligurica (K. Daniel, 1903), Oreonebria (Oreonebria) angusticollis (Bonelli,

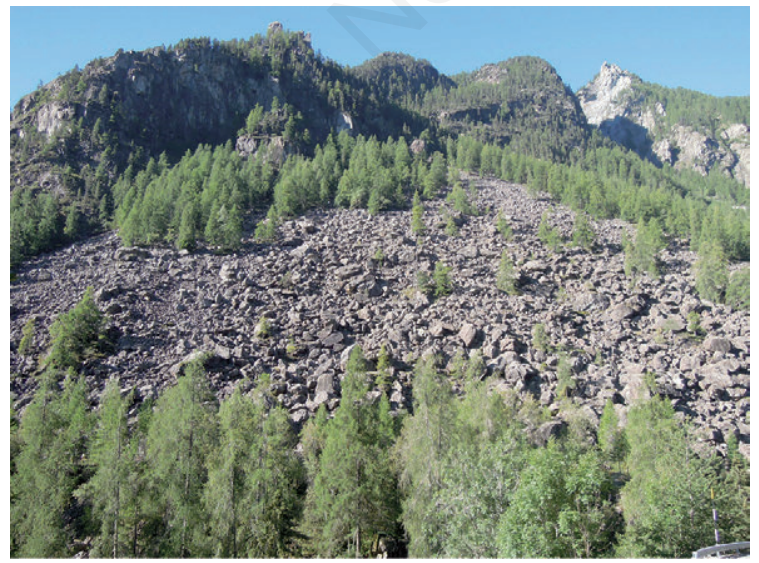

Fig. 1. Paleofrana a grandi blocchi nei pressi di Outre-l'Éve (Valle di Champorcher), sede di una popolazione di Oreonebria gagates (Foto Luigi Bisio, 4.IX.2017).
1810) e Nebria (Nebriola) cordicollis kochi Schatzmayr, 1939 (Fig. 2). La scomparsa più o meno rapida della copertura nevosa, con la precoce xericità superficiale che ne consegue, condiziona in modo marcato la fenologia di questa entità all'esterno che è perciò molto precoce e contratta. Durante il breve periodo di comparsa all'esterno la specie viene predata dai ragni che vivono sulla superficie del macereto, come testimonia la presenza di elitre nelle tele degli stessi osservata in alcune stazioni (cfr. Bisio, 1998).

Molto più efficaci sono invece le ricerche con l'impiego di trappole innescate con aceto (addizionato con acido salicilico a scopo conservante). Date però le caratteristiche del substrato roccioso, non sempre è possibile utilizzare le classiche trappole a caduta (pitfall traps) costituite da bicchieri di plastica da interrare verticalmente con l'apertura verso l'alto al livello del piano di campagna. Si possono sostituire con bottiglie-trappola da introdurre all'interno delle fenditure e delle piccole cavità tra i blocchi di roccia, $\mathrm{o}$, meglio ancora (se ciò è possibile), all'interno di piccole grotte che talora si aprono tra i massi accavallati, soprattutto se questi sono di dimensioni ciclopiche. Ne è un esempio la grande cavità, già descritta in passato dall'autore, alla base di un'estesa conoide detritica situata nel Vallone di Sea (Valli di Lanzo) (cfr. Bisio, 1998). Anche gli esemplari che lo scrivente ha rinvenuto di recente nella Valle di Champorcher, nei pressi dell'abitato di Outre-l'Éve (Champorcher), sono stati catturati

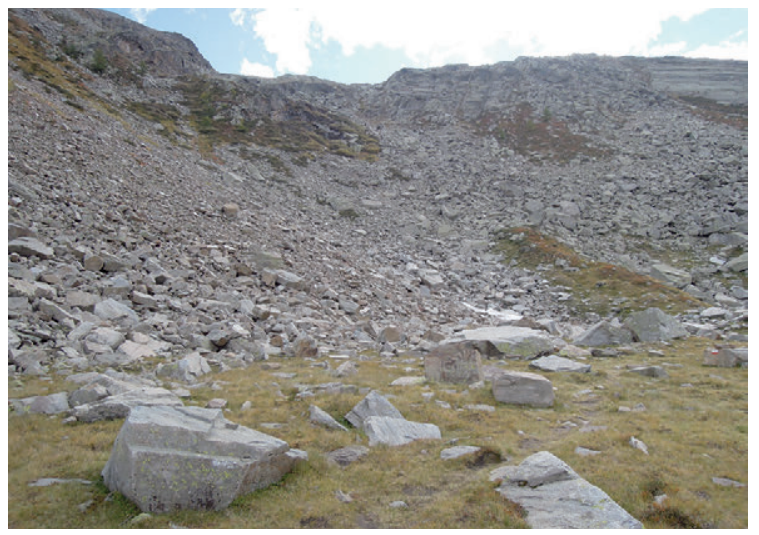

Fig. 2. Pian di Nel (Valle Orco): macereti a grandi blocchi all'interno dei quali è stata osservata la presenza di Oreonebria gagates in sintopia con Nebria cordicollis e Oreonebria angusticollis (Foto Luigi Bisio, 24.IX.2017). 
con lo stesso metodo all'interno di una piccola grotta che si apre tra i blocchi di roccia di un'enorme paleofrana (Figg. 3 e 4). Le bottiglie-trappola si possono collocare all'interno delle fenditure, in posizione inclinata sulla superficie dei blocchi stessi, avendo cura di appoggiarne il collo su materiale vario (pietre, ghiaia o muschio) reperito in loco, allo scopo rendere accessibile l'imboccatura della bottiglia agli insetti. Questa metodologia di ricerca ha permesso di rivelare che nelle zone più profonde del macereto la fenologia della specie risulta molto più prolungata, probabilmente favorita dalle basse temperature pressoché costanti che caratterizzano tali zone anche nei periodi più caldi dell'estate. Nella grotta della Valle di Champorcher, ad esempio, gli esemplari sono stati censiti in un periodo compreso tra la fine di maggio e l'inizio di settembre, cioè il periodo più caldo e secco della torrida estate del 2017. O. gagates è stata rinvenuta anche nelle zone più profonde di grotte più grandi ( $\mathrm{cfr}$. Ghiliani, 1887; Sturani, 1978; Lana et al., 2004; Casale \& Giachino, 2010).

Nei macereti compresi nella fascia forestale e in quella arbustiva la specie risulta spesso sintopica con Sphodropsis ghilianii ghilianii (Schaum, 1858), elemento ipogeofilo molto comune in questi litosuoli (cfr. Bisio, 1998). In tali ambienti, inoltre, non è infrequente la presenza di popolazioni di Oreonebria (Oreonebria) castanea castanea (Bonelli, 1810), specie perinivale nella fascia alpina che risulta presente in sede microclasica in quella forestale dove fa registrare depressioni altimetriche importanti (la specie è stata osservata a soli $1200 \mathrm{~m}$ nel macereto della Valle di Champorcher).

O. gagates è stata anche osservata come specie ripicola nei torrentelli in foresta o in biotopi sorgivi nella fascia alpina (cf. Binaghi, 1951: sub pedemontana; Bisio, 1986, 1998; Coulon, 1994; Focarile, 1975) dove, a causa del suo peculiare praeferendum igrotermico, essa vive spesso semisommersa nelle fessure tra i sassi. È peraltro da segnalare una vistosa eccezione a questa abitudine, relativa ad alcuni esemplari raccolti dallo scrivente in un torrentello poco a valle dell'Alpe Ciabraressa in Val Pellice (Bisio, 2004). Il primo individuo della specie è stato visto correre sul greto in pieno sole ed è stato scambiato a prima vista per una più comune Nebria (Eunebria) jockischii jockischii Sturm, 1815. Riconosciutolo come appartenente alla specie in oggetto immediatamente dopo la cattura, lo scrivente ne trovava altri quattro individui sotto i sassi lungo le rive. Un ulteriore individuo veniva poi ancora rinvenuto circa un mese dopo. Poiché ricerche effettuate nello stesso luogo in anni successivi sono state del tutto infrut-

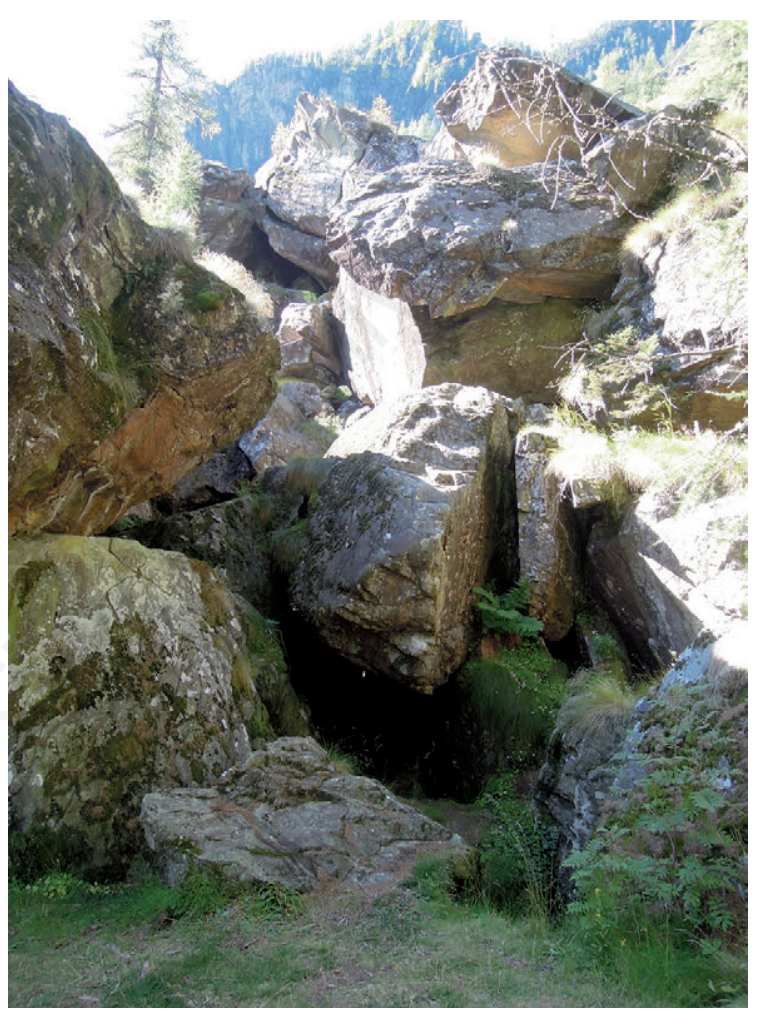

Fig. 3. Outre-l'Éve (Champorcher): ingresso della grotta tra i massi della paleofrana (foto Luigi Bisio, 4.IX.2017).

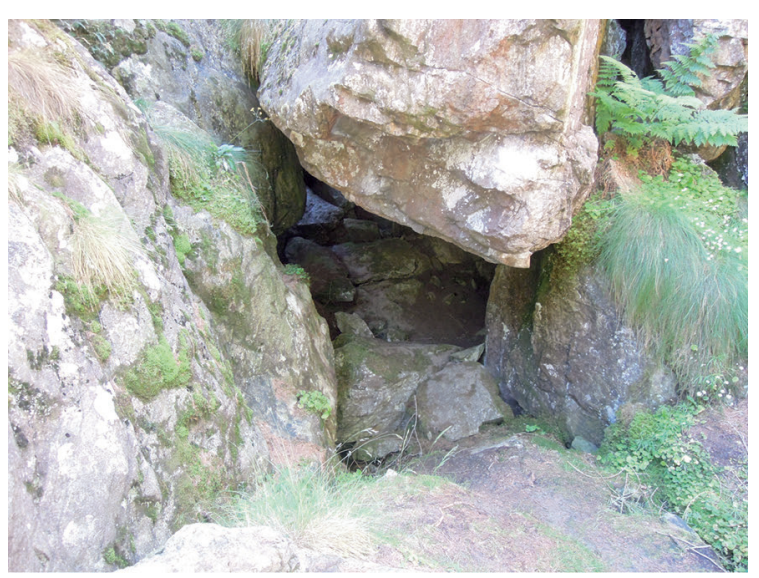

Fig. 4. Outre-l'Éve (Champorcher): particolare dell'ingresso della grotta (foto Luigi Bisio, 4.IX.2017). 
tuose, non è da escludere che si trattasse di individui presenti accidentalmente, forse fluitati da una piena. Nei torrentelli e nei biotopi sorgivi $O$. gagates è stata osservata talvolta in sintopia con Nebria (Nebriola) laticollis Dejean, 1826 (cfr. Focarile, 1975; Bisio, 1998), elemento ripicolo stenotermo caratterizzato da un'altrettanto spiccata frigofilia e anch'esso legato di conseguenza ad acque molto fredde.

Forse a causa delle sue esigenze igrotermiche $O$. gagates si rinviene più di frequente nelle valli interessate da apporti meteorici consistenti, valli delle quali si conoscono in effetti un maggior numero di stazioni (v. oltre) e, talvolta, anche popolazioni relativamente più numerose, quali la Valle Po, la Val Pellice, la bassa Valle Orco, la Val Chiusella e la Valle di Champorcher. A fronte di un maggior numero di dati relativi a queste valli fa riscontro l'apparente maggiore sporadicità della specie in valli nelle quali il tasso di precipitazioni è decisamente inferiore, quali la Val Chisone e la Val di Susa, sporadicità che sembra confermata, tra l'altro, anche dagli esiti negativi dei campionamenti con l'impiego di trappole a caduta eseguiti a cura del Parco Orsiera-Rocciavrè (cfr. Allegro \& Viterbi, 2010).

La specie presenta un excursus altitudinale piuttosto ampio che abbraccia i piani montano, subalpino e alpino e che è compreso tra 1200 e $2600 \mathrm{~m}$. Il limite inferiore assoluto è stato riscontrato di recente nella Valle di Champorcher, ma la specie è stata rinvenuta a quote molto basse anche in Val Chiusella $(1300 \mathrm{~m})$; in entrambi i casi si tratta di depressioni altimetriche estreme forse favorite dal clima piovoso che caratterizza queste due valli. In altre valli, secondo quanto risulta dalle indagini svolte, la specie non sembra quasi mai scendere al di sotto dei $1500 \mathrm{~m}$. Sulla base di queste considerazioni, pare ragionevolmente certo che le segnalazioni relativa a Viù (Daniel \& Daniel, 1891; Magistretti, 1965) e Fenestrelle (Bänninger, 1924) siano da ritenersi riferite ai monti circostanti e non alla quota specifica dei due centri abitati (rispettivamente 785 e $1145 \mathrm{~m}$ ).

Invece, per quanto concerne le quote più elevate, $i$ dati a disposizione sono ancora frammentari e in gran parte derivati da reperti casuali, poiché, per motivi logistici, non è sempre possibile utilizzare il metodo d'indagine con l'uso di trappole in zone d'alta quota che sono, già di per sé, accessibili con difficoltà. Sotto questo aspetto, i recenti campionamenti con l'impiego di trappole a caduta operati di recente a cura del Parco del Gran Paradiso cominciano a certificare la presenza frequente della specie anche nella fascia alpina. Pertanto, il limite superiore attualmente noto di $2600 \mathrm{~m}$, registrato in tre sole stazioni situate rispettivamente nel vallon de la Grande Sassière, in Val Pel-

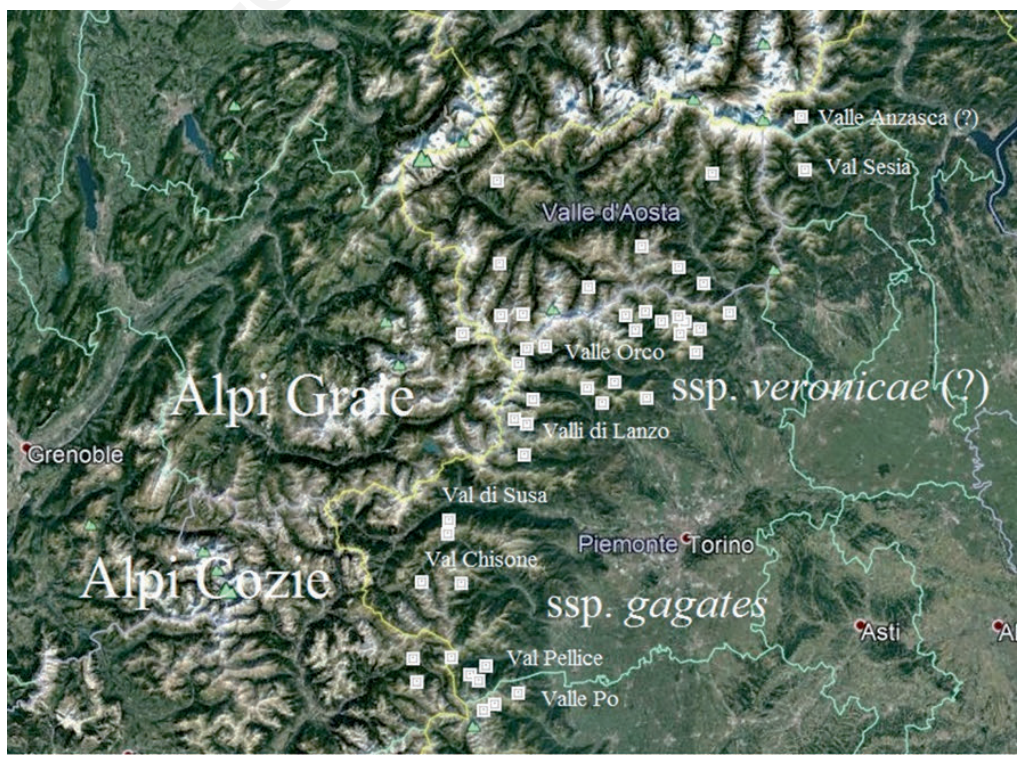

Fig. 5. Corologia di Oreonebria gagates. 
lice e in Valgrisenche, potrebbe in futuro essere suscettibile di incremento.

\section{Corologia}

O. gagates è a tutt'oggi nota delle seguenti stazioni (Fig. 5).

Per ogni località riporto la citazione bibliografica, il raccoglitore e chi me l'ha segnalata, se diverso dal raccoglitore ( $\mathrm{JC}=$ Jacques Coulon; LB = Luigi Bisio; PNGP=Parco Nazionale del Gran Paradiso; RM=Riccardo Monguzzi).

Francia: Hautes-Alpes (Provence-Alpes-Côted'Azur): Abriès (Jeannel, 1941); Abriès, 1 es. collezione Porta (Focarile, 1975); vallon de Ségure (Ristolas) m 1700 (JC). Savoie (Rhône-Alpes): vallon de la Grande Sassière (Tignes) m 2600, 14 es. in varie date (ssp. veronicae sensu Coulon, 1994).

Italia: Piemonte CN: Valle Po: «Je l'ai trouvée en bien petit nombre dans le voisinage du Mont-Viso» (Ghiliani, 1847: sub Nebria lafresnayei); Grotta di Rio Martino (Crissolo) (Ghiliani, 1887: sub Nebria gagates); Monviso (Ganglbauer, 1891: sub Nebria baudii); Crissolo, 1 es., Baudi di Selve leg. (Focarile, 1975); ibidem a m 1500, 1 es. V.1980 (Bisio, 1986); Pian della Regina (Crissolo) m 1800, pl. es. in varie date (Bisio, 1986); 1251 Pi/CN, "Pertui de l'Oustanetto" (Ostana) VIII.2003, Lana leg. (Lana et al., 2004). TO: Val Pellice: Alpe Ciabraressa (Comba dei Carbonieri) m 1700, 5 es. 28.V.2001, 1 es. 27.VI.2001 (Bisio, 2004); Grange della Gianna (Comba dei Carbonieri) m 1800, 3 es. 17.V.1992 (Bisio, 1998); Col Barant (Comba dei Carbonieri) m 2200, 1 es. 2.VII.1997, Allegro leg. (Bisio, 2004); Col Boucie (Villanova, Bobbio Pellice) m 2600, 2 es. 15.VII.1979 (Bisio, 1986). Val Germanasca: Pian delle Sagne (Vallone del Pis, Massello) m 2100, 1 es. 23.VIII. 1997 (Bisio, 1998). Val Chisone: Fenestrelle, 1 es. VIII.1871, Baudi di Selve leg. (Focarile, 1975); Fenestrelle, 1 es. VII.1893 (Bänninger, 1924: sub Nebria pedemontana); Monte Albergian (Souchères Basses, Pragelato) $\mathrm{m}$ 1700, Giachino leg. (Bisio, 1986). Val di Susa: Colle delle Finestre, 1 es. VIII.1941, Rocca leg. (Focarile, 1975). Valli di Lanzo: «rara nelle alte Valli di Lanzo.» (Baudi di Selve, 1890: sub Nebria pedemontana); Viù (? v. oltre) (Daniel \& Daniel, 1891); Balme (Daniel \& Daniel, 1891: sub Nebria pedemontana; Bänninger, 1924: sub Nebria pedemontana); Pian della Mussa m 1700, 1 es. 19.V.1993 (Bisio, 1998); Vallone di Sea
(Forno Alpi Graie) m 1500, pl. es. (trappole) 6.VI./9.IX.1991, 12.V./7.IX.1992. (Bisio, 1998); Santuario della Madonna di Ciavanis (Vallone del Colle della Paglia, Chialamberto) m 1900, 7 es. 10.VI.1996 (Bisio, 1998); ibidem, 3 es. 2.VI.2001 (LB); Lago di Monastero (Cantoira) m 2000, 27.VI.1987, Giuntelli leg. (Bisio, 1998). Valle Orco: Vallone Gallenca, Pian della Pessa (Monte Soglio, Canischio) m 1500, 5 es. (trappole) 4.V./7.IX.1990 (Bisio, 1998); Alpe Cialma (Monte La Cialma, Locana) m 1800, 3 es. (trappole) 11.V./8.IX.1992 (Bisio, 1998); Ceresole Reale, Born leg. (Bänninger, 1924: sub Nebria pedemontana); Pian di Nel (dintorni Rifugio Jervis, Ceresole Reale) m 2300, 2 es. 7.VII.1983 (Bisio, 1986); Gran Piano di Noasca m 2200 (pitfall traps) (Allegro \& Viterbi, 2009). Val Soana: Grange Vassinetto (Boschietto, Vallone di Forzo), m 2050, 4 es. 5.VI.1996 (Bisio, 1998); Campiglia Soana-San Besso m 2000 e m 2200 (pitfall traps) (Allegro \& Viterbi, 2009); Grange Santanel (Piamprato), m 1800, 2 es. (trappole) 5.V/5.IX.1993 (Bisio, 1998); Grange d'Arlens (Pianetto) m 1800, 11 es. (trappole) 10.V/13.IX.1988 e 7.V/5.IX.1989 (Bisio, 1998). Valle Sacra: Vallone Piova, Alpe Valossera (Punta Quinzeina-MonteVerzel, Castelnuovo Nigra): 1500-1650, 2 es. (trappole) 28.IV./12.IX.1994, 4 es. e 4 paia di elitre 13.V.1996 (Bisio, 1998). Val Chiusella: Valpiana (Vallone Savenca Monte Verzel, Issiglio) m 1800, 2 es. (trappole) 10.IV./5.IX.1995 (Bisio, 1998); Alpe Vaudanza (Seggiovie Palit, Delpizzen) $\mathrm{m}$ 1300-1350, 2 es. (trappole) 7.V./8.IX.1995, 3 es. 7.V.1995, 7 es. 6.V.1996 (Bisio, 1998); mulattiera per l'Alpe Pasqua (Fondo-Tallorno) m 13001 es. (trappole) 13.IV./18.IX.1992 (Bisio, 1998). Canavese nord-orientale: $1609 \mathrm{Pi} / \mathrm{To}$, Grotta del Ghiaccio della Cavallaria $\mathrm{m}$ 1553, 2 es. 2.VIII.1942, 1 es. 14.VIII.1942 (Sturani, 1978); ibidem, 20.IX.2003, Giachino \& Lana leg. (Lana et al., 2004; Casale \& Giachino, 2010); Cima Bonze, 1 es. (RM). VC: Val Sesia: Cima Tre Croci (Val Vogna, Riva Valdobbia) $m$ 2200, Locca leg. (Bisio, 1998). VB: Valle Anzasca: Alpe Rosareccio (Macugnaga) (Stabile, 1853: sub Alpaeus foudrasi e sub Alpaeus lafresnayei; Favre, 1890: sub Nebria foudrasi e sub Nebria lafresnayei); Alpe Pedriola (Stabile, 1853: sub Alpaeus lafresnayei. Valle d'Aosta (AO): imbocco della Valle d'Aosta: Becca di Renon (Donnas, Clapey) versante NE, 1 es. VI.1979, Focarile leg. (Bisio, 1986). Valle di Champorcher: Outre-l'Éve m 1200, 7 es. 31.V./3.VII.2017, 6 es. 3.VII./5.IX.2017 (LB). Val Chalamy: Monte Barbe- 
ston m 2200, 1 es. (Focarile, 1976). Valle di Cogne: Ozein m 1430-1550, «un modesto numero di esemplari» (Focarile, 1975). Valsavarenche: Orvieille m 2400 (pitfall traps) (PNGP). Valle di Rhêmes: Vaudalettaz m 2200 (pitfall traps) (Allegro \& Viterbi, 2009). Valgrisanche: Becca del Merlo, versante N (Arvier) m 2600, un paio di elitre VII-1978, Focarile leg. (Bisio, 1986). Val Sapin (Courmayeur): Trou-des-Romains (miniera abbandonata) m 1714, VIII-1931, Doria leg in collezione Monguzzi (Bisio, 1986). Val d'Ayas: Champoluc, in collezione dell'Istituto di Entomologia dell'Università di Torino, Casale det. (Bisio, 1986).

Lungo il versante francese, quindi, la specie è ancora nota solo di pochissime stazioni distanti tra di loro e il suo areale complessivo è di fatto ancora in gran parte sconosciuto.

Lungo il versante italiano, invece, l'areale della specie pare non discostarsi da quello delineato nei miei precedenti lavori (Bisio, 1986, 1998). Il limite meridionale è ormai identificato nel versante Nord del Monviso (Valle Po), come sembrano confermare gli esiti negativi di una serie di indagini svolte dallo scrivente con l'impiego di trappole più a Sud in Val Varaita, Val Maira e Val Grana in biotopi apparentemente favorevoli all'insediamento della specie. Quello settentrionale sarebbe invece rappresentato dalle stazioni della Valle Anzasca sulla base di alcune vecchie segnalazioni di Stabile (1857: sub Alpaeus foudrasi e sub Alpaeus lafresnayei), in parte riprese da Favre (1890: sub Nebria foudrasi e sub Nebria lafresnayei). Sull'esattezza di tali segnalazioni qualche dubbio continua peraltro ancora a sussistere, non tanto per il relativo isolamento delle stazioni citate da Stabile rispetto al resto dell'areale: non si può infatti escludere che le lacune molto ampie che ancora oggi sussistono tra il solco della Valle d'Aosta e la Valle Anzasca (malgrado la zona sia stata esplorata da generazioni di entomologi) possano essere solo la conseguenza di una oggettiva rarefazione delle popolazioni della specie, come talora si riscontra per molti taxa ai margini del loro areale. È un fatto, a tal proposito, che, mentre quasi ogni tentativo di ricerca con l'impiego di trappole effettuato nei macereti delle valli canavesane ha consentito di rilevare la presenza della specie, ricerche simili condotte per anni in Val d'Ayas (dove pure la specie risulta presente) e nella Valle di Gressoney (le cui caratteristiche climatiche parrebbero molto favorevoli alla presenza di $O$. gagates) hanno avuto esiti del tutto negativi. Del resto la recente segnalazione per la Val Sesia parrebbe rendere un po' più verosimili le segnalazioni di Stabile. Ciò che invece lascia piuttosto dubbiosi sull'esattezza delle segnalazioni di questo autore è la relativa abbondanza di dati che egli sarebbe stato in grado di ottenere nei dintorni di Macugnaga, considerando la ben nota oggettiva difficoltà di ottenere reperti della specie con semplici ricerche a vista: egli infatti segnalava «Alpaeus foudrasi» dell'Alpe Rosareccio e "Alpaeus lafresnayei» dell'Alpe Rosareccio e dell'Alpe Pedriola, quindi due specie raccolte in tre stazioni, il che fa presumere anche un buon numero di esemplari raccolti, situazione che pare alquanto improbabile per i motivi anzidetti. È inoltre da considerare che l'autore, tra le specie raccolte nei dintorni di Macugnaga, non citava Oreonebria (Oreonebria) picea (Dejean, 1826), entità che nella valle è molto comune. Poiché nella zona del Monte Rosa non sono infrequenti esemplari di grandi dimensioni di quest'ultima specie che potrebbero essere facilmente scambiati a prima vista per individui di $O$. gagates, non si può neanche escludere l'ipotesi di un errore di determinazione da parte dell'autore.

Per quanto riguarda altre zone del suo areale italiano, è soprattutto nelle Alpi Graie, tra la destra orografica della Valle d'Aosta e la Val Grande di Lanzo, che $O$. gagates pare presentare popolazioni più continue e meno sporadiche censite quasi tutte con l'impiego di trappole, mentre sembra nuovamente rarefarsi a Sud di quest'ultima valle. In effetti, ricerche approfondite effettuate dallo scrivente con analoga metodologia nelle Valli d'Ala e di Viù, così come in diverse valli delle Alpi Cozie, sono state del tutto infruttuose. Sono significativi in tal senso gli esiti del tutto negativi di ricerche trentennali della specie con l'impiego di trappole in Val Pellice, valle per la quale le poche stazioni note sono il risultato di reperti del tutto casuali ottenuti in un territorio che lo scrivente ha frequentato assiduamente e minuziosamente per molti anni.

\section{TASSONOMIA E SISTEMATICA}

O. gagates fu descritta da Bonelli (1810: sub Alpaeus gagates) su materiale tipico del quale non si conosce con sicurezza la provenienza, poiché l'autore non ne riportò in modo preciso la località di raccolta, ma si limitò solo ad affermare che la specie «habite les Alpes».

La specie venne successivamente ridescritta da Vuillefroy (1868-1869) col nome di Nebria pedemon- 
tana sulla base di un unico esemplare proveniente dal Piemonte («je l'ai reçu sans autre indication») che precisava l'autore - «présente la forme générale de la $N$. Lafresnayei [ $=$ Nebria (Nebria) lafresnayei Serville, 1821]».

A causa della sua apparente rarità e del conseguente ridotto numero di esemplari presenti nelle collezioni, O. gagates fu in seguito oggetto di dubbi e di interpretazioni difformi, spesso controverse, per quanto concerne sia il suo status tassonomico, sia la sua collocazione sistematica. Che nell'Ottocento le idee fossero tutt'altro che chiare lo dimostrano anche i lavori dei piemontesi Ghiliani e Baudi di Selve. Il primo autore (Ghiliani, 1847) citava tra i Coleotteri del Piemonte a lui noti «Nebria Lafresnayei (N. gagates Bonelli)», sottintendendo in tal modo una sinonimia; nel suo successivo «Elenco delle specie di Coleotteri trovate in Piemonte» (Ghiliani, 1887) egli citava invece separatamente «gagates Bon.», «Lafresnayei Serv.», «Foudrasi Dej..» [=Nebria (Nebria) lafresnayei foudrasi Dejean, 1828] e pedemontana, trattate quindi tutte come specie distinte. Baudi di Selve (1890) ritenne di correggere in parte tale opinione; infatti, pur mantenendo pedemontana come specie distinta, riteneva invece foudrasi «una leggera varietà» di lafresnayei e riprendeva la sinonimia «gagates Bon... = Lafresnayei Serv.», peraltro in contrasto con le regole della priorità.

L'impossibilità di esaminare gli esemplari tipici e di confrontarli quindi tra loro - nel frattempo infatti quelli di Bonelli erano stati distrutti dagli antreni, mentre la collezione di Vuillefroy era stata divisa e quindi l'unico esemplare era stato disperso (cfr. K. Daniel \& J. Daniel, 1891: p. 49) -, così come le notizie alquanto generiche sulla loro reale provenienza, non consentirono a molti autori successivi di chiarire la questione del reale status tassonomico di $O$. gagates e della sua posizione sistematica.

Infatti, i fratelli Daniel (K. Daniel \& J. Daniel, 1891), i primi a tentare di risolvere la questione, dopo aver esaminato alcuni esemplari della collezione Baudi di Selve considerarono gagates e pedemontana due specie distinte, la prima simile a Nebria (Nebria) hellwigii (Panzer, 1797) e a Nebria (Nebria) lafresnayei Serville, 1821 e la seconda affine alle specie del gruppo di $O$. castanea. Inoltre, pur non avendo potuto prendere visione degli esemplari tipici, ne ipotizzarono la provenienza da Viù nelle Valli di Lanzo («Bonelli’s einziges Stück wurde indes von Peiroleri bei Viù (Valle di Stura) in den grajischen Alpen gesammelt»). Occorre però rilevare che un'attenta lettura del lavoro di Bonelli rende piuttosto dubbia questa ipotesi che forse è l'esito di una disattenzione o di un'interpretazione errata del testo. Come già evidenziato, Bonelli (1810) non citò nessuna località precisa di provenienza, mentre la frase «Cette espèce .... été trouvée par Mr. Peyroleri dans la vallée de Viù sous des pierres.» (che i fratelli Daniel sembrano ricalcare a grandi linee nel loro lavoro) è riferita invece a O. castanea, descritta da Bonelli (sub Alpaeus castaneus) subito di seguito a $O$. gagates.

Contemporaneamente ai fratelli Daniel, Ganglbauer (1891) ridescrisse la specie col nome di $\mathrm{Ne}$ bria baudii, pur facendo trasparire qualche dubbio su di una sua possibile identità sia con gagates ( $\ll$ ?gagates Bon.»), sia con pedemontana ( $\ll$ ?pedemontana Vuillefr.»). In effetti tra la fine dell'Ottocento e la prima metà del Novecento si ignorava cosa fosse realmente «gagates Bon.», come dimostra il fatto che, più di vent'anni dopo, Fiori (1913) la segnalasse del Passo del Melogno, del Passo dei Giovetti, di Caprauna e della Val Pesio, segnalazioni che sono certamente errate e da riferire a esemplari di Nebria (Nebria) tibialis tibialis (Bonelli, 1810). La segnalazione relativa al Passo del Melogno venne poi ripresa anche da Luigioni (1929).

Non molto diverso, rispetto a quello proposto dai fratelli Daniel, fu il quadro tassonomico e sistematico delineato da Bänninger (1924): N. pedemontana specie distinta da inserire nel subg. Oreonebria, che era stato istituito poco più di vent'anni prima da $\mathrm{K}$. Daniel (1903) per O. castanea, Nebria baudii sinonimo junior di $N$. pedemontana e, in totale contrasto con le regole della priorità, $N$. gagates $=N$. lafresnayei, a sua volta considerata un Alpaeus. Il quadro delineato da questo autore diventò probabilmente l'opinione corrente dell'epoca e fu ripreso tale e quale da Luigioni (1929), da Porta (1923) e da Winkler (19241932). Anche Junk (1927), nel suo «Coleopterorum Catalogus», mantenne la stessa posizione, ma, se non altro, corresse la sinonimia in $N$. lafresnayei $=N$. gagates, rispettando la priorità di quest'ultima.

A far luce finalmente sull'argomento fu Jeannel (1941) che stabilì per lafresnayei lo status di specie distinta e per pedemontana quello di sinonimo junior di gagates, indicando per quest'ultima «mont Viso» come locus typicus. Inoltre, l'autore stesso, che precedentemente (Jeannel, 1937) aveva elevato al rango generico il subg. Oreonebria, ascrisse gagates a questo genere, 
istituendo per essa il subg Nebriorites (Jeannel, 1941).

Nonostante i chiarimenti forniti da Jeannel, Magistretti (1965) si limitò a riportare le opinioni contrastanti degli autori precedenti, affermando che la questione pedemontana-gagates-lafresnayei sembra insolubile a causa dell'impossibilità di confrontare le serie tipiche.

Successivamente, Focarile (1975), pur concordando sulla distinzione specifica di Nebria lafresnayei e sulla sinonimia di $N$. pedemontana con gagates proposta da Jeannel, ha criticato la collocazione sistematica di quest'ultima, ritenendo più corretto considerarla affine alla stessa $N$. lafresnayei e quindi da trattare come una Nebria in senso stretto piuttosto che una Nebria del subg. Oreonebria (l'autore infatti ha retrocesso Oreonebria al rango subgenerico).

La collocazione sistematica di gagates proposta da Focarile, in un primo tempo condivisa da diversi autori della scuola italiana (Bisio, 1986, 1998; Casale \& Vigna Taglianti, 1993; Vigna Taglianti, 1993), è stata però poi abbandonata (cfr. Vigna Taglianti, 2005; Pesarini \& Monzini, 2010) e si è tornati considerare la specie come una Oreonebria (nel frattempo il subg. Oreonebria era stato elevato nuovamente al rango di genere). Invece, la scuola francese, da subito, non ha condiviso l'opinione di Focarile ed è rimasta fedele alla sistematica proposta da Jeannel (1941) (cfr. anche Bonadona, 1971; Coulon, 1994; Coulon et al., 2000, 2011; Ledoux \& Roux, 2005). Anche altri autori europei hanno accettato quest'ultima opinione (cfr. Löbl \& Smetana, 2003).

Coulon (1994), sulla base di 14 esemplari raccolti nel vallon de la Grande Sassière, ha descritto, per quella che egli ha continuato appunto a chiamare Oreonebria gagates, la sottospecie veronicae. I caratteri in base ai quali la sottospecie si distinguerebbe da quella nominale si possono così riassumere:

- pronoto meno largo e meno arrotondato nella parte anteriore con lati sinuati davanti agli angoli posteriori che sono nettamente acuti e sporgenti in fuori (Fig. 6). Chetotassi del pronoto comprendente 5-6 setole disposte solo lungo il margine della metà anteriore. Lobo mediano dell'edeago con base non rettilinea e curvatura basale più pronunciata. Paramero destro più lungo e non troncato all'estremità (ssp. gagates);

- pronoto più largo e più arrotondato nella parte anteriore con lati non sinuati e convergenti regolarmente verso gli angoli posteriori che sono meno acuti e non sporgenti in fuori (Fig. 7). Chetotassi del pronoto comprendente 10-13 setole disposte lungo tutto il margine. Lobo mediano dell'edeago con base rettilinea e curvatura basale meno pronunciata. Paramero destro più breve e troncato all'estremità (ssp. veronicae).

Successivamente, Coulon et al. (2000, 2011), sulla base della fotografia e della descrizione pubblicate da Focarile (1975) per gli esemplari della Valle di Cogne, hanno ipotizzato di attribuire le popolazioni valdostane a questa sottospecie. In effetti, pur sulla base del numero ridotto di esemplari che lo scrivente ha potuto esaminare, non si può negare che gli esemplari provenienti dalle Alpi Graie (tra la destra orogra-

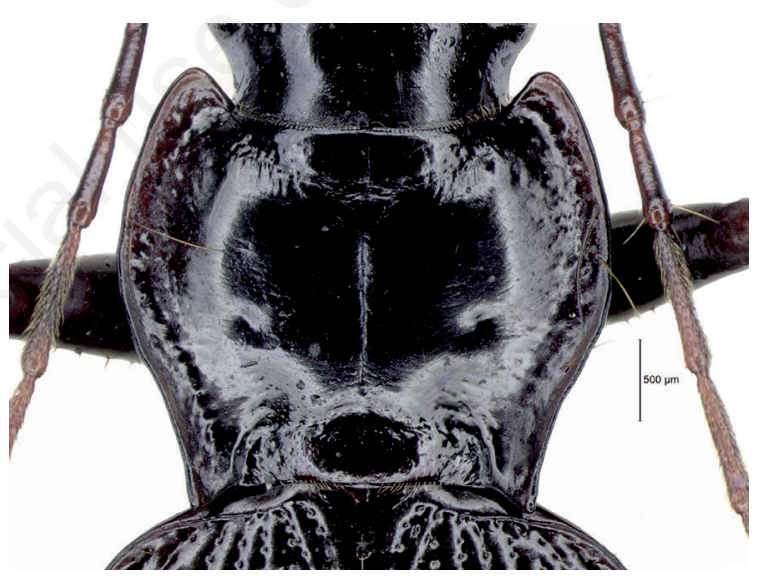

Fig. 6. Pronoto di Oreonebria gagates del Col Barant (Val Pellice) (foto Gianni Allegro).

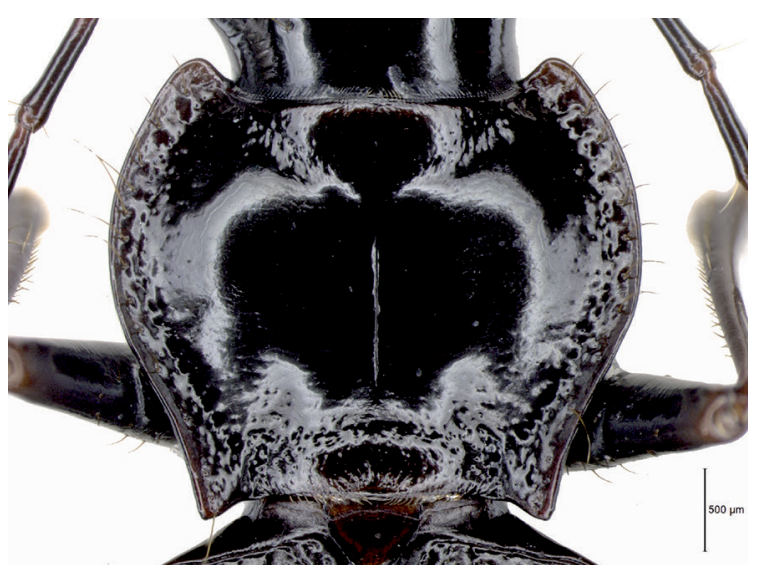

Fig. 7. Pronoto di Oreonebria gagates del Gran Piano di Noasca (Valle Orco) (foto Gianni Allegro). 
fica della Valle d'Aosta e la Val Grande di Lanzo) presentino tutti i caratteri diagnostici descritti per la ssp. veronicae e che i pochissimi delle Alpi Cozie siano invece riconducibili alla ssp. nominale. Occorre però rilevare che la costanza di tali caratteri dovrebbe comunque essere verificata con l'esame di un gruppo ben più congruo di esemplari di quello attualmente disponibile e, soprattutto, formato da individui provenienti da tutte le diverse zone dell'areale della specie, cosa tuttavia non facile da realizzare a causa delle ampie lacune che ancora caratterizzano tale areale. Limitando il discorso al versante italiano dal quale proviene il maggior numero di esemplari noti, della zona centrale dell'areale (cioè di una zona che comprende la Val Germanasca, la Val Chisone, la Val di Susa, la Val Sangone, la Valle di Viù e la Val d'Ala) è nota soltanto una decina di esemplari. Di fatto è quindi attualmente impossibile stabilire se i caratteri diagnostici sopra elencati siano variabili in senso clinale (e quindi se la specie sia monotipica) o se esista effettivamente una netta separazione tra la forma delle Alpi Cozie e quella delle Graie, tale da giustificare la validità della ssp. veronicae. Tuttavia, pur non potendo assumere un'opinione netta su una delle due ipotesi, sotto l'aspetto climatico la seconda non parrebbe da scartare del tutto: infatti, non si può escludere che il solco della Val di Susa, con il suo clima xerico poco favorevole per questa specie dalla spiccata igrofilia (come del resto dimostra il fatto che a tutt'oggi di questa valle sia noto un unico esemplare proveniente dal Colle delle Finestre), possa aver costituito a tutti gli effetti una barriera invalicabile, tale da isolare nettamente le due popolazioni e favorirne la conseguente differenziazione.

\section{RINGRAZIAMENTI}

Desidero ringraziare tutti coloro - amici, colleghi e famigliari - che, nell'arco di più di trent'anni, hanno contribuito a diverso titolo alla raccolta di questi dati e alla loro elaborazione. Si ringraziano inoltre il Prof. Jacques Coulon per la comunicazione di dati corologici relativi al territorio francese, Ramona Viterbi e il Parco Nazionale Gran Paradiso per la concessione di dati inediti, Gianni Allegro per la prima lettura del manoscritto e per l'esecuzione delle fotografie dei pronoti. Un ringraziamento particolare va infine all'amico Achille Casale per la revisione critica del manoscritto.

\section{BIBLIOGRAFIA}

Allegro G.,Viterbi R., 2009 - Note faunistiche ed ecologiche sui carabidi del Parco Nazionale del Gran Paradiso (Coleoptera Carabidae). Rivista Piemontese di Storia Naturale, 30: 129-147.

Allegro G., Viterbi R., 2010 - Contributo alla conoscenza faunistica ed ecologica dei Carabidi del Parco Naturale Orsiera Rocciavrè e della Riserva di Foresto (Coleoptera, Carabidae). Rivista Piemontese di Storia Naturale, 31: 187-212.

BÄNNINGER M., 1924 - Di alcune specie di Nebria e Leistus della regione mediterranea ( $8^{\circ}$ Contributo alla conoscenza dei Carabini, Col.). Bollettino della Società Entomologica Italiana, 56: 97-106.

Baudi Di Selve F., 1890 - Catalogo dei Coleotteri del Piemonte. Annali Regia Accademia Agricoltura Torino, 32 (1889): 51274.

BINAGHI G., 1951 - Coleotteri d'Italia. Vita, ambienti, utilità, danni, mezzi di lotta. Briano, Genova, 210 pp.

Bisıo L., 1986 - Nebria gagates Bonelli specie interessante del Piemonte e della Valle d'Aosta: nuovi reperti (Coleoptera Carabidae). Rivista Piemontese di Storia Naturale, 7: 107-112.

Bisio L., 1998 - Note aggiuntive su Nebria gagates e su alcune specie della carabidofauna associata (Coleoptera Carabidae). Rivista Piemontese di Storia Naturale, 18 (1997): 205-232.

Bisıo L., 2004 - Note sui Coleotteri Carabidi dell'alta e media Val Pellice (Alpi Cozie) e osservazioni sulla carabidofauna della Conca del Prà (alta Val Pellice) (Coleoptera Carabidae). Rivista Piemontese di Storia Naturale, 25: 283-329.

Bonadona P., 1971 - Catalogue des Coléoptères Carabiques de France. Nouvelle revue d'Entomologie, Supplément, 1: 1-177.

Bonelli F. A., 1810 - Observations entomologiques. I. Mémoires de l'Académie impérial de Science, Turin, 18: 21-78.

Brandmayr P., Colombetta G., Drovenik B., Forti E., Poldini L., Brandmayr Zetto T., 1980 - Étude multidisciplinaire sur l'écologie de quelques Carabides endogés (Coleoptera Carabidae). Mémoires de Biospéologie, 7: 85-98.

Casale A., Giachino P.M., 2010 - Due nuovi Coleotteri ipogei delle Alpi Occidentali: Duvalius (Duvalius) lanai n. sp. (Carabidae: Trechini) e Archeoboldoria sturanii n. sp. (Cholevidae: Leptodirinae). Rivista Piemontese di Storia Naturale, 31: 213-240.

Casale A.,Vigna Taglianti A., 1993 - I Coleotteri Carabidi delle Alpi occidentali e centro-occidentali (Coleoptera Carabidae). Biogeographia, Lavori Società Italiana di Biogeografia, (n. s.), 16 (1992): 331-399. 
Coulon J., 1994 - Description préliminaire d'une nouvelle Oreonebria de Haute-Tarentaise (Savoie), O. gagates veronicae n. ssp. (Coleoptera, Nebriidae). Bulletin de la Société entomologique de France, 99 (1): 105-106.

Coulon J., Marchal P., Pupier R., Richoux P., Allemand R., Genest L.C., Clary J., 2000 - Coléoptères de Rhône-Alpes, Carabiques et Cicindèles. Société linnéenne de Lyon, 193 pp.

Coulon J., Pupier R., QuÉInnec E., Ollivier E., Richoux P., 2011 - Coléoptères Carabiques. Compléments aux deux volumes de René Jeannel, mises à jour, correction et répertoire. Faune de France 94 (vol. 1): 1-352, planches I-XII.

DANIEL K., 1903 - Bestimmungtabellen der europäische Koleopteren LII (Nebriini, Notiophilini, Trachypachyni, Epactrini, Elaphrini und Lorocerini). Münchener Koleopterologische Zeitschrift, 1 (1902/1903): 155-173.

Daniel K., Daniel J., 1891 - Beiträge zur Kenntnis der Gattung Nebria Latr. I. Vier neue Arten aus Piemont und den Abruzzen. II. Ueber Nebria gagates Bonelli und pedemontana Vuillefroy. Coleopteren Studien, München, 1: 41-53.

DeJEAn P., 1828 - Species général des Coléoptères de la collection de M. le Comte Dejean. Tome troisième. Paris, MéquignonMarvis, VII + 556 pp.

FAVRE E. 1890 - Faune des Coléoptères du Valais et des régions limitrophes. Nouvelles Mémoires de la Société Helvetique des Sciences Naturelles 31, 448 pp.

FIORI A., 1913 - Studio sopra alcune specie dei gen. Leistus e Nebria. Rivista Coleotterologica Italiana, 11 (9-11): $182-201$.

FoCARILE A., 1973 - Sulla Coleotterofauna alticola del Gran San Bernardo (versante valdostano). Annali della Facoltà di Agraria dell'Universita di Torino, 9: 51-118.

FoCARILE A., 1975 - Alcuni interessanti Coleotteri della Valle d'Aosta. Revue Valdôtaine d'Histoire Naturelle, 29 : 8-52.

FoCARILE A., 1976 - Sulla Coleotterofauna alticola del Monte Barbeston m 2482 (Val Chalamy) e del Monte Nery m 3076 (Val d'Ayas). Revue Valdôtaine d'Histoire Naturelle, 30: 86-125.

Ganglbauer, L., 1891 - Die Käfer von Mitteleuropa. 1. Band, Familienreihe Caraboidea. Carl Gerold's Sohn, Wien, 557 pp.

Ghiliani V., 1847 - Mémoire sur la station de quelques coléoptères dans les différentes régions du Piémont. Annales de la Société Entomologique de France, 5: 83-142.

Ghiliani V., 1887 - Elenco delle specie di Coleotteri trovate in Piemonte (opera postuma a cura di L. Camerano). Annali Regia Accademia Agricoltura Torino, 29 (1886): 195-381.

Giachino P.M., VAILATI D., 2016 - Riflessioni sulla terminologia biospeleologica: i concetti di troglobio, troglofilo e troglosseno. Atti del Convegno: "La Ricerca Carsologica in Italia. Frabosa Soprana (Cn)", 22-23 giugno 2013. Laboratorio carsologico sotterraneo di Bossea, Frabosa Soprana, 195-200.

JEANNEL R., 1937- Notes sur les Carabiques. Revue française d'Entomologie, 4: 1-23.

Jeannel R., 1941 - Coléoptères Carabiques I (Faune de France, vol. 39). P. Lechevalier (Paris), 1-571.

JunK W. 1927 - Colepterorum Catalogus. Pars 92: Carabidae, Carabinae, Trib. Nebriini. 5: 341-390.

Lana E., Casale A., Giachino P.M., 2004 - Attività biospeleologica 2003. Grotte, Bollettino del Gruppo Speleologico Piemontese, GSP CAI-UGET, Torino,, 47 (142): 36-41.

Ledoux R., Roux P., 2005 - Nebria (Coleoptera, Nebriidae). Faune Mondiale. Muséum Société linnéenne de Lyon, 976 pp.

LöBl I., Smetana A. (eds.), 2003 - Catalogue of Palaearctic Coleoptera. I. Archostemata-Myxophaga-Adephaga. Apollo Books, Stenstrup, $819 \mathrm{pp}$.

Luigioni P., 1929 - I Coleotteri d'Italia. Catalogo sinonimico-topografico-bibliografico. Memorie della Pontificia Accademia delle Scienze "I nuovi Lincei", Roma, 13: 1-1160.

Magistretti M., 1965 - Fauna d'Italia, VIII. Coleoptera: Cicindelidae, Carabidae. Catalogo topografico. Calderini, Bologna, $512 \mathrm{pp}$.

Pesarini C., Monzini V., 2010 - Insetti della Fauna Italiana. Coleotteri Carabidi I. Milano, 152 pp.

PorTA A., 1923 - Fauna Coleopterorum Italica. I. Adephaga. Piacenza, 285 pp.

StABILE J., 1853 - Des Coléoptères observés au Mt-Rose, val Macugnaga. Actes de la Société Helvétique des Sciences Naturelles, 38: 214-222.

SturAni M., 1978 - La Grotta del Ghiaccio (pubblicazione postuma a cura di A. Casale). Grotte, Bollettino del Gruppo Speleologico Piemontese, GSP-CAI UGET, 21, (65): 9-10.

Vigna Taglianti A., 1993 - Coleoptera Archostemata Adephaga I (Carabidae). In: Minelli A., Ruffo S. \& La Posta S. (eds.), Checklist delle specie della fauna italiana, 44. Edizioni Calderini, Bologna, $51 \mathrm{pp}$.

Vigna Taglianti A., 2004 - Fauna Europaea: Carabidae. In: Audisio P. (ed.), 2004. Coleoptera 2. Fauna Europaea version 1.1. Available from: http://www.faunaeur.org.

Vigna Taglianti A., 2005 - Checklist e corotipi delle specie di Carabidae della fauna italiana. Appendice B. pp. 186-225. In: P. Brandmayr, T. Zetto \& R. Pizzolotto (eds.), I Coleotteri Carabidi per la valutazione ambientale e la conservazione della biodiversità. Manuale operativo. APAT, Manuali e Linee Guida, 34: 240 pp.

Vuillefroy F., 1868-1869 - Insectes nouveaux de la Faune Européenne. L'Abeille, 5: 289-295.

WinKLER A., 1924-1932 - Catalogus Coleopterorum regionis palaearcticae. WINKLER A. ed., Wien, 1698 pp. 